\title{
Métamorphose des organisations rurales au Cameroun
}

Implications pour la recherche-développement et la gestion des

ressources naturelles

\section{The transformation of rural organizations in Cameroon}

Implications for research and development and the

management of natural resources

\section{Philippe-René Oyono et Ludovic Temple}

\section{Numéro 288, avril 2003}

URI : https://id.erudit.org/iderudit/1022201ar

DOI : https://doi.org/10.7202/1022201ar

Aller au sommaire du numéro

\section{Éditeur(s)}

Institut de l'économie sociale (IES)

\section{ISSN}

1626-1682 (imprimé)

2261-2599 (numérique)

\section{Découvrir la revue}

\section{Citer cet article}

Oyono, P.-R. \& Temple, L. (2003). Métamorphose des organisations rurales au Cameroun : implications pour la recherche-développement et la gestion des ressources naturelles. Revue internationale de l'économie sociale, (288), 68-79. https://doi.org/10.7202/1022201ar

\section{Résumé de l'article}

La réforme rurale conduite au Cameroun depuis 1992 a introduit de nouveaux schémas de fonctionnement dans l'infrastructure communautaire agricole et la gestion locale des forêts. Il s'en est suivi une prolifération d'organisations rurales, regroupées sous l'étiquette de "groupes d'initiative commune " (GIC), de « nouvelles coopératives » et de " comités de gestion des forêts ». Cet article décrit l'organisation communautaire en milieu rural camerounais et propose une première évaluation des changements institutionnels et organisationnels qui ont marqué les années 90. Les auteurs analysent les contraintes véhiculées par ce modèle de réforme rurale, en termes de fonctionnalité, de couverture efficiente des espaces géographiques et d'adéquation aux problématiques de développement rural. Ils montrent aussi que ces formes d'organisation communautaire constituent des opportunités importantes pour la recherche agronomique, le développement agricole et la gestion locale des ressources forestières. 


\title{
MÉTAMORPHOSE DES ORGANISATIONS RURALES AU CAMEROUN
}

\author{
Implications pour la recherche-développement
}

et la gestion des ressources naturelles

(*) Sociologue. Center for inter national forestry research (Cifor), bureau régional pour l'Afrique centrale et de 1'ouest, BP 2008, Yaoundé-Messa, Cameroun. Mél. : r.oyono@ogiar.org.

(**) Economiste. Centre interna tional de recherche agronomique pour le développement (Cirad), Centre africain de recherche sur le bananier plantain (Carbap), BP 2572, Yaoundé.

Vél. : 1.temple@carmet.cm par Philippe-René Oyono (") et Ludovic Temple ${ }^{(*)}$

La réforme rurale conduite au Cameroun depuis 1992 a introduit de nouveaux schémas de fonctionnement dans l'infrastructure communautaire agricole et la gestion locale des forêts. Il s'en est suivi une prolifération d'organisations rurales, regroupées sous l'étiquette de "groupes d'initiative commune" (GIC), de "nouvelles coopératives " et de "comités de gestion des forêts ». Cet article décrit l'organisation communautaire en milieu rural camerounais et propose une première évaluation des changements institutionnels et organisationnels qui ont marqué les années 90. Les auteurs analysent les contraintes vébiculées par ce modèle de réforme rurale, en termes de fonctionnalité, de couverture efficiente des espaces géographiques et d'adéquation aux problématiques de développement rural. Ils montrent aussi que ces formes d'organisation communautaire constituent des opportunités importantes pour la recherche agronomique, le développement agricole et la gestion locale des ressources forestières.

es échecs des formes traditionnelles d'appui au développement rural T ont conduit à l'émergence d'approches participatives. En Afrique subJsaharienne, ces approches exercent depuis dix ans une influence notable sur le développement rural, la recherche agronomique et la gestion des ressources naturelles (Ssenkoloto, 1984, pp. 107-120; Mondjanagui, 1984 pp. 23-30). Les soubassements théoriques et opérationnels du modèle participatif prennent forme dans de nouveaux paradigmes tels le "renforcement de la société civile " (Lachenman, 1984, pp. 62-35), le "désengagement de l'Etat" (Mercoiret, 1990, pp. 49-58) et la "gouvernance " (Amalric, 1994, pp. 14-18). Plus que la participation en soi, le débat cristallise généralement des divergences sur les modalités de sa production et sur les formes de son exercice en tant qu'instrument de praxis sociale et de changement (Selener, 1997, pp. 204-220). Dans une large mesure, les travaux empiriques portant sur le contenu social du développement agricole en milieu rural des pays sub-sahariens révèlent que la participation ainsi conceptualisée et idéalisée est populaire et communautaire, et non individuelle. L'ajustement de la participation au communautarisme est ainsi déterminé par l'organisation collective. Au Cameroun, celle-ci a toujours été mise 
(1) Cet article est le produit d'un travail de recherche réalisé : àl'In . temational Institute of tropical agriculture (Cameroun), sur "Organizational forms, rural orga nizations and NGOS in Southern Cameroon : institutional linkages in support of rural development » au Centre international de recherche agronomique pour le développement, dans une action thématique programmée, «L'ap provisionnement alimentaire des villes : les organisations au cour des ajustements ».

(2) Ces années sont marquées par des évolutions décisives dans le pay sage sociopolitique du Cameroun, à l'instar de la lépalisation de la liberté d'association (1990), de la légali. sation du pluralisme politique (1990), de la réforme rurale (1992) et de la promulgation d'une légis lation forestière "décentralisée 》 (1994). à contribution dans la recherche du bien-être collectif en milieu rural (Goyette et Kom, 1993, pp. 2-4). Dans l'ensemble, elle a subi de nombreuses transformations (Oyono, 2001, pp. 14-20). Le présent article ${ }^{(1)}$ tente de documenter les mutations organisationnelles qui ont affecté le milieu rural camerounais ces dernières années. Il met ainsi en exergue les conditions d'émergence de ces organisations ${ }^{(2)}$ et questionne leur adéquation aux problématiques de recherche et de développement. En dernière instance, l'article isole les implications de ces évolutions sur, d'une part, les perspectives de mise en œuvre de nouvelles « méthodologies » sociales pour la recherche agricole et, d'autre part, les options de développement agricole. En somme, la présente contribution tente d'expliciter la thèse selon laquelle l'innovation politique et les réformes institutionnelles ont des effets ambivalents sur le développement agricole et la gestion des forêts en milieu rural camerounais. En effet, au moment où ces changements introduisent de nouvelles dynamiques, ils engendrent aussi des contre-effets comme la mise sous tutelle des organisations rurales et leur instrumentalisation externe.

\section{Le contexte d'émergence des transformations organisationnelles}

(3) La liste des associations his toriques dans les ethnies du Cameroun ne peut être reproduite ici. Nous ne prendrons à cet effet que quelques exemples.
L'analyse de l'organisation collective mise en place pour assurer le bien-être humain en milieu rural camerounais fait ressortir trois périodes essentielles, elles-mêmes divisées en sous-périodes. Dans la période précoloniale, les formes d'organisation sociale reposaient sur les systèmes ancestraux de régulation des interactions humaines, des pratiques sociales et des représentations collectives, comme l'ont développé Alexandre (1965, pp. 17-26), Russel (1993, pp. 7-10 et 19-21) et Diaw (1997, pp. 12-14). A cet égard, outre l'autorité lignagère, une pluralité de cercles sociaux modélisaient les comportements " holistiques " à l'intérieur des communautés (agrégats rituels, sociétés initiatiques, sociétés secrètes, organisations culturelles et organisations socio-économiques).

\section{Archéologie des organisations rurales}

Dans cette évolution, les organisations rurales prenaient des formes diverses, que l'on peut regrouper dans le concept générique « mise en commun des acteurs sociaux ». Ces dynamiques associatives - ouvertes à tous les acteurs sociaux - étaient, de par leurs éléments fondateurs, polymorphes. Rentraient, à titre d'exemples, dans ces formations grégaires ${ }^{(3)}$ les ako chez les Banyang (province du Sud-Ouest), les mendzogro chez les Ewondo (province du Centre), les bishuala chez les Kwassio (province du Sud), les nséele chez les Banganté (province de l'Ouest), etc. En fait, chaque ethnie articulait des dispositifs sociaux, coopératifs et récréatifs destinés à classer et à arranger l'effort social pour le bien-être collectif.

La période coloniale, à travers l'administration et la chrétienté, inaugure la dilution progressive des organisations « historiques » dans de nouvelles figures 
(4) Beaudoux (1994, pp. 30-32) reproduit les axes majeurs de ces évolutions. Le gouvernement a mis en place des recommandations menées avec 1 'appui d'institutions intemationales; il a fait voter des lois au Parlement ; il a pris des décrets d'application et a mis en place des procédures d'enregis trement des organisations rurales par provinces. et de nouveaux schémas. Dans l'espace rural, les transformations qui affectent la société traditionnelle et les éclatements organisationnels en question donnent naissance à des dynamiques moins connectées au substrat social. C'est le cas, dans la deuxième période, de l'émergence officielle des « associations ", des " unions " et des " company " (concept tiré d'un argot appelé pidgin english), agrégats plus " opératifs" et portés vers la résolution de problèmes communautaires. Ces constructions se sont superposées aux précédentes.

La troisième période est représentée par les trois premières décades de la période postcoloniale. Des déterminants étatiques - ministère de l'Agriculture, organismes para-étatiques, ministère de l'Administration territoriale - suscitent une nouvelle fois une métamorphose des organisations rurales. Des coopératives étatiques, des groupements d'agriculteurs modernes (GAM), des comités villageois de développement (CVD), des villages councils (dans la partie anglophone du Cameroun) se surimpriment sur de nombreux schémas organisationnels existants. Ces processus sont déjà une première illustration de la mise sous tutelle de la paysannerie camerounaise (Geshiere, 1984, pp. 13-33) et mettent en avant la forte implication des intervenants extérieurs dans la problématique du développement rural.

\section{La réforme rurale et la reconstruction organisationnelle}

Au début des années 90, le Cameroun, comme beaucoup d'autres pays d'Afrique, recompose la trame institutionnelle des rapports entre l'Etat et la société civile. C'est ainsi que la loi sur la liberté d'association est promulguée en 1990, suivie de la loi no 92/006 de 1992 sur les groupes d'initiative commune et les coopératives. Ces législations successives se sont chargées de porter la réforme rurale camerounaise ${ }^{(4)}$, sous la dictée méthodologique d'une structure créée par le Bureau international du travail, la Central Unit for rural organization's reform (Curor). Du coup, une rente financière internationale est mise en circulation par la Banque mondiale pour soutenir la réforme: c'est dans ces circonstances que le Fonds d'intervention pour les micro-réalisations agricoles et communautaires (Fimac) est ébauché. Le programme Fimac, exécuté sur l'ensemble du territoire de 1992 à 1998, a permis de financer de nombreuses micro-réalisations agricoles au niveau des GIC, notamment pour l'achat du petit équipement (agricole, pastoral et piscicole), à travers des ONG locales.

Dans la province du Centre, 200 GIC ont été touchés par le Fimac de 1992 à 1997 pour des financements estimés au total à 370000 USD. Dans le seul département de Nyong et So’o (province du Centre), 64 GIC ont reçu des financements pour environ 100000 USD. L'Union européenne et la Caisse française de développement, en soutien, créent à leur tour le Fonds d'appui aux organisations rurales (Fondaor) qui, de son côté, finance des programmes de formation des responsables des GIC à la gestion administrative et à la gestion comptable. La vaste initiative conjointe Cameroun-Banque mondiale appelée Programme national de vulgarisation et de recherche agricole (PNVRA), dont les principaux bénéficiaires sont les GIC, constitue également une implication positive de la réforme rurale. 
De nombreux autres vecteurs provoquent, parfois dans la précipitation et dans l'ignorance, le changement organisationnel. En plus des organismes comme la Société de développement du cacao (Sodecao), des projets de coopération et des ONG, des sous-préfets en tournée dans les villages et des politiciens en campagne électorale - sans prévisibilité raisonnable exhortent les paysans à créer des "groupes " pour l'amélioration de leurs conditions de vie. Une palette d'opérations a accompagné cette refonte du paysage organisationnel en milieu rural camerounais: phagocytose, superposition, recyclage, mise sous tutelle partielle, adhésion opportuniste, etc. De la conjugaison de tous ces facteurs surgissent, au milieu des années 90, une prolifération et une inflation sans précédent d'organisations rurales.

Les métamorphoses organisationnelles obtenues configurent, dans l'ensemble, trois types d'organisations: des GIC à l'intérieur des villages (organisations dites de premier niveau); des unions de GIC (organisations de deuxième niveau) à l'échelle trans-villageoise; et, à l'échelle régionale, des fédérations des unions de GIC (organisations de troisième niveau). Rien que dans la province du Centre du Cameroun, l'étude qui a fourni une partie du matériau de cet article a recensé 14 unions de GIC regroupant 462 GIC répartis dans 59 villages. La naissance en masse des GIC s'effectue soit sur les décombres d'organisations villageoises déliquescentes, soit sur des organisations existantes, mais qui doivent se faire " reconnaître officiellement " par les services du ministère de l'Agriculture, soit, enfin, à partir de rien.

\section{Nombre d'organisations rurales répertoriées dans le département} de Nyong et So'o (province du Centre) de 1994 à 1998

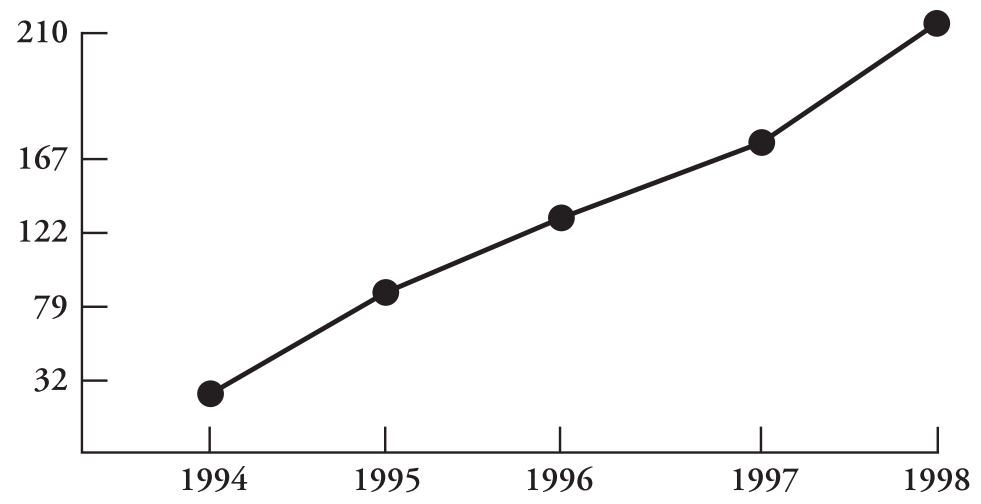

Source: Fimac-Mbalmayo 
(5) C'est ainsi que l'on trouve des « GIC palmier à huile », « GIC maïs ", " GIC banane », avec des signifi cations comme "L'union fait la force », « Tous pour un », « Ami cale », etc.

(6) Voir note 5.
L'idée sous-jacente à la démarche paysanne de création des GIC est de «se mettre ensemble » et de fédérer les efforts socio-économiques en en orientant le potentiel vers la production économique et l'amélioration des revenus. Au départ, les volontaires pour la constitution de l'organisation se réunissent et lui assignent des objectifs. Une dénomination lui est par la suite attribuée ${ }^{(5)}$. La phase de structuration des GIC comprend deux étapes, le renforcement du processus d'adhésion et l'élaboration des documents organiques (statuts et règlements intérieurs). L'élaboration des documents organiques constitue la condition sine qua non de demande de l'enregistrement officiel (et de la reconnaissance juridique). Une fois enregistré, le GIC peut prétendre accéder à des sources de financement externes. Dans la réalité, cet accès est souvent conditionné par le parrainage des ONG.

La dénomination des GIC rend explicite tant leurs choix « opératifs » que leur connotation socioculturelle ${ }^{(6)}$. Ladhésion, régie par la proximité sociale, est négociée dans le terreau des liens familiaux et villageois. Le décryptage de la composition des membres d'une union de GIC, Solidarité pour le développement de la zone Akak-Melan (province du Centre), a montré que $80 \%$ des groupes disposent d'une base sociale essentiellement familiale. Les objectifs proclamés des GIC se situent en droite ligne des domaines d'activité (spécialisation agricole, pisciculture, épargne-crédit, formation, information). Les règlements intérieurs de ces organisations établissent des dispositifs institutionnels reposant sur les droits et devoirs des membres, identifiés par l'étude comme étant les seuls garants de l'action collective. Ainsi, le référentiel « GIC » recouvre et « habille » des organisations très diverses, aux fonctions hétéroclites: c'est une nébuleuse. Il s'ensuit une certaine confusion dans l'analyse des capacités de ces organisations à constituer un relais durable au désengagement de l'Etat et dans l'appui au développement agricole principalement. Si l'un des éléments à l'origine de l'instabilité des GIC est, comme nous l'avons vu, le flou dans les motivations de leurs promoteurs et leur diversité, la reconnaissance de cette diversité implique des travaux supplémentaires qui pourraient aboutir à une évolution vers des cadres juridiques plus adaptés.

\section{Les limites des organisations issues de la réforme}

\section{Les limites fonctionnelles}

Ce contexte qui a favorisé l'émergence des GIC a, parallèlement, facilité la construction de liens opérationnels et stratégiques avec une multitude d'acteurs. Les ONG et les projets de coopération se situent en première ligne de ces acteurs. Dans l'ensemble, chaque GIC - et chaque union de GIC - est connecté à une ONG ou à un projet. Les perspectives d'adoption et d'amélioration des technologies agricoles ont élargi cet espace de collaboration à la recherche conventionnelle et à la diffusion. L'explosion organisationnelle générée par la réforme du monde rural a été un mouvement 
à effet d'indétermination à bien des égards. De nombreux GIC se transforment en constructions circonstancielles, issues parfois du « paternalisme " des ONG et des projets, dans un élan réducteur et parfois infantilisant. Selon les membres de beaucoup de GIC interviewés, les demandes d'appui (ou de financement) n'émanent pas très souvent des bénéficiaires eux-mêmes: ce sont les ONG qui en imposent généralement le contenu. Dans plusieurs cas, la négociation n'est effectuée qu'avec les responsables du GIC. L'interprétation des propos et des «non-dits » des promoteurs des GIC montre que collecter au passage des ressources financières (subventions et micro-crédits) reste la visée majeure dans de nombreux cas. C'est la raison pour laquelle plusieurs GIC, en proie à des enjeux " alimentaires ", ont disparu.

Il s'ensuit que de nombreux GIC sont frappés de paralysie. Trente pour cent des 310 GIC interviewés lors de l'étude demeurent à cet effet des structures précaires. Sur un tout autre plan, il est de plus en plus admis que la tradition communautaire qui supporterait les organisations paysannes est un «mythe » (Oyono, 2001, pp. 40-63). En fait, ce ne sont pas des constructions monolithiques. Elles obéissent à des contradictions, à des différenciations internes et à des conflits organisés sur le contrôle des ressources financières captées. Les enseignements ci-dessus, déduits de la phénoménologie des GIC, montrent que les changements organisationnels issus de la réforme rurale sont susceptibles d'hypothéquer le développement agricole escompté. Par ailleurs, le fonctionnement « théâtral » des GIC et les motivations financières qui les gouvernent ne peuvent qu'infléchir négativement les options de partenariat durable avec la recherche et l'appui au développement. Il convient d'ajouter à ces facteurs limitants la tendance qu'ont les promoteurs à utiliser les GIC comme des ressources sociopolitiques et des vecteurs d'ascension individuelle.

\section{Les conflits de " parrainage "}

L'analyse des conditions d'émergence des organisations de producteurs ou " organisations rurales " à travers la formalisation juridique révèle

\section{Les GIC, quelle réalité?}

"Beaucoup de GIC ont été créés de façon opportuniste par des ONG, le Fimac et des sous-préfets. Leurs promoteurs voulaient accéder à des crédits: quand ce fut fait, ces organisations sont devenues des coquilles vides. Lorsquelles n'ont rien eu, elles ont continué à attendre, en fonctionnant superficiellement. Dans les villages, les réunions sont convoquées par les présidents [des $\mathrm{GIC}]$ : ce sont eux qui connaissent l'ordre du jour, le reste des membres sont des accompagnateurs, ils font le nombre."

Source: Louis Monewosso, agronome, Centre pour le développement autocentré (Cedac), Sangmélima, province du Sud, le 13 mars 1998. 
une dynamique issue de la convergence de trois facteurs principaux: le retrait de l'Etat, couplé avec l'ouverture de voies institutionnelles permettant de légitimer les formes d'organisation paysanne; les incitations données par les bailleurs de fonds, qui transitent le plus souvent par des ONG disposant de stratégies de captage des financements (Engola, 1997); le refoulement collectif des préoccupations réellement agricoles. La réalité socio-économique des GIC est donc très éclatée et fluide: elle explique, en grande partie, leur faiblesse "programmatique " et leur incapacité à durer ou à s'approprier des fonctions économiques. Il est à noter que, si les ONG accompagnent le processus d'émergence ou de formalisation des GIC, ce processus aboutit parfois à des ruptures (conflit entre des ONG et la Fédération des organisations paysannes du Nyong et Mfoumou, par exemple). En effet, les organisations ayant acquis une certaine maturité analysent très vite le fait d'être récupérées pour des raisons stratégiques par des $\mathrm{ONG}$ en quête de financements extérieurs et qui veulent, selon l'expression consacrée, "s'enrichir sur le dos des paysans".

\section{Les limites morpho-structurales}

En complément des GIC, au premier niveau de l'organisation communautaire des producteurs ruraux (niveau intra-villageois), se multiplient de manière croissante des unions de GIC (niveau trans-villageois) et des fédérations de GIC (niveau régional), qui tentent d'institutionnaliser, sur le plan politique, les dynamiques en cours. Le fonctionnement de ces unions ou de ces fédérations reste très complexe lorsque l'on tente de les mettre en relation avec la multiplicité des fonctions qu'elles assument. L'étude d'une fédération de GIC localisée dans la province du Centre (la Fopanym) révèle un emboîtement de plusieurs niveaux d'intervention et une inextricabilité de sa structuration géographique. Le fonctionnement des organisations nouvelles rencontre des difficultés liées à leur structuration et à leur morphologie.

Le maintien d'une pluralité de fonctions conduit à des difficultés importantes, notamment dans la gestion de ces organisations. Par ailleurs, la reconnaissance par l'Etat de ces nouveaux interlocuteurs est un élément nécessaire à une structuration du développement rural: "Les dynamiques paysannes demandent, pour naître et se consolider, des conditions favorables qui dépendent en grande partie de l'Etat" (Mercoiret, 1990). Cette reconnaissance par l'Etat n'est pas évidente au Cameroun. Les organisations de deuxième et de troisième niveau sont instables dans le modèle camerounais de réforme rurale, au regard de leurs conditions d'émergence et compte tenu des attitudes encore prudentes développées à leur égard par l'Etat et les bailleurs de fonds. L'orientation d'un certain nombre d'entre elles vers des objectifs de commercialisation en commun des produits agricoles (Moustier, 1997) est vraisemblablement, à l'heure actuelle, un élément structurant de leur développement - mais non unique. Cette stratégie rencontre de 
nombreuses contraintes déterminées par la construction progressive de dispositifs cognitifs de coordination pour la définition de règles de fonctionnement communes. Ce processus doit s'appuyer sur des apprentissages nouveaux. En effet, la structuration de ces règles sur des territoires englobant une diversité de formes sociales de coordination, liées notamment à la pluralité des appartenances à des lignages et à des communautés linguistiques et ethniques, constitue une difficulté supplémentaire.

\section{La Fédération des organisations paysannes du Nyong et Mfoumou (Fopanym)}

La Fopanym coordonne les activités de 19 unions de GIC qui concernent 2500 adhérents. Elle a bénéficié à l'origine d'un financement de lancement par l'intermédiaire d'une ONG. Des dissensions ont conduit ensuite à une rupture. La structure est dotée d'un local. Le financement est issu de plusieurs sources: des cotisations directes d'une centaine d'adhérents qui en contrepartie bénéficient de l'avance à crédit en intrants; des prélèvements (environ $25 \mathrm{FCFA} / \mathrm{kg}$ ) sur des opérations de commercialisation en gros du café ou du cacao en regroupant la production des unions de GIC ou des GIC isolés; des subventions de fonctionnement. L'emboîtement de plusieurs niveaux d'organisation est lié aux difficultés de financement de la structure et à l'instabilité des unités de base (GIC). La fédération, de manière récente, s'implique dans la commercialisation des produits vivriers (macabo, plantain). Dans un premier temps, au niveau d'une union de GIC, les producteurs s'informent sur les quantités qu'ils pourraient commercialiser individuellement. Le délégué (coordinateur) de l'union centralise ces informations lorsqu' il atteint un tonnage suffisant pour remplir un camion de 6 tonnes; il part ensuite négocier à Yaoundé $(100 \mathrm{~km})$ la location d'un camion. Le prix de la transaction varie entre 120000 et 180000 francs CFA, toutes charges comprises. Il contacte alors la fédération et demande une avance de 100000 francs CFA; l'union, de son côté, avance 60000 francs CFA. Une fois l'accord établi, le délégué se rend sur les marchés destinataires pour s'informer des prix. Il retourne ensuite informer les membres de l'organisation des conditions dans lesquelles va se réaliser l'opération. Le jour convenu, le transporteur, accompagné du délégué, collecte et charge la production.

Les adhérents de la fédération participant à l'opération s'interdisent de vendre aux bayams salams (revendeuses) de passage. Cette pression sociale est donc tributaire des éléments de cohésion entre adhérents. Elle est principalement induite par les fonctions qu'assure la fédération, notamment l'approvisionnement en crédits, mais également l'approvisionnement en médicaments de première nécessité (nivaquine et aspirine). La réalisation de ces fonctions est l'élément principal de cohésion. Deux ou trois producteurs sont délégués pour aller vendre. Les producteurs négocient directement la vente de leurs produits avec les demi-grossistes sur le marché destinataire. La coordination entre producteurs membres de la fédération se matérialise notamment sur l'homogénéisation dans la configuration des lots. Après la réalisation de l'opération, les représentants des unions remboursent à la Fopanym l'avance de 100000 francs CFA. Il lui reste un solde qu'il divise entre les producteurs au prorata des quantités amenées. De nombreuses limites apparaissent dans le fonctionnement de la fédération: l'absence de contrôle démocratique de la gestion; le manque de formation en gestion financière; l'extraordinaire diversité des GIC quant aux raisons qui ont présidé à leur existence; la réticence des ONG dans l'accompagnement de ces niveaux d'organisation "supérieurs ». 


\section{Configuration de la diversification organisationnelle dans le Cameroun rural}

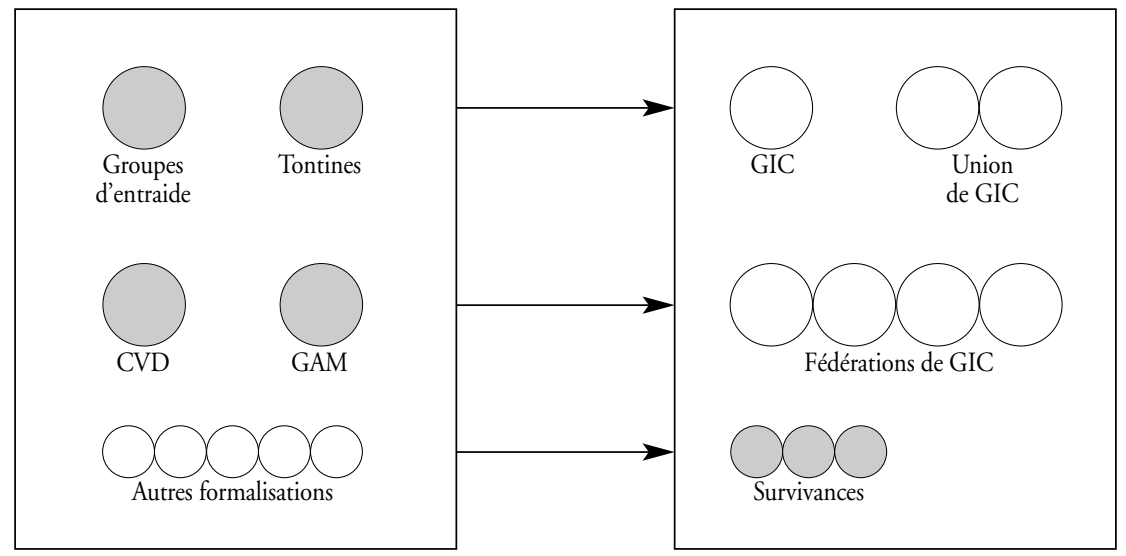

CVD : comités villageois de développenent.

GAM : groupements d'agriculteurs modernes.

La référence à des territoires en mutation perpétuelle dans la recombinaison sociale (permanence forte des dynamiques migratoires) rend difficile la mobilisation des règles de cohésion sociale anciennes construites sur une histoire culturelle commune. Il devient alors nécessaire de reconstruire ces règles à partir de nouvelles formes de légitimité sociale et économique. L'amélioration des rapports de négociation lors de la mise sur le marché des produits en est une. Elle établit et renforce un processus de coopération pour la recherche classique d'économies d'échelle, pour augmenter l'efficacité ou les rendements des activités de commercialisation (le stockage du cacao, le transport des produits vivriers et l'accroissement des pouvoirs de négociation vis-à-vis des autres opérateurs). Dans cette évolution, certaines organisations de type unions de GIC ou fédérations de GIC sont naturellement appelées à évoluer vers des formes de coopérative. L'évolution vers ce type d'organisation implique cependant de stabiliser des formes de gestion interne flexibles. En l'occurrence, des exemples pris dans des réalités sociales différentes montrent que les modalités de rémunération des adhérents sont au centre de l'efficacité des coopératives et de leur capacité à renforcer le pouvoir de négociation des producteurs sur les différents marchés (Jarrige et Touzard, 2001). L'instabilité actuelle des organisations rurales peut s'expliquer par leur professionnalisation naissante. La maîtrise progressive de leur environnement institutionnel devrait permettre de diminuer cette instabilité et de renforcer leur rôle dans l'animation du développement rural et l'organisation d'interfaces fonctionnelles entre tous les acteurs concernés. 


\section{Implications pour la recherche-développement et la gestion des ressources naturelles}

(7) Il s'agit de persomnes lettrées, ayant voyagé et plus ouvertes aux innovations.
Pour répondre aux demandes des bénéficiaires, la recherche conventionnelle a, depuis une vingtaine d'années, opéré un déplacement de certaines de ses priorités en s'impliquant, par exemple, dans l'élaboration de plates-formes partenariales (Hartman, 1987, pp. 62-69; Thrupp, 1996, pp. 10-23). Le partenariat avec les organisations rurales et les ONG est né de cette exigence. Au Cameroun, l'installation d'une dynamique de complémentarité avec la recherche et l'intervention libère depuis peu des transactions, certes timides, mais prometteuses, avec le nouveau paysage organisationnel. La récession économique dans laquelle le Cameroun s'est plongé à la fin des années 80 a induit l'épuisement du modèle urbain. C'est dans ce contexte que la tendance à l'exode rural s'est significativement estompée, pour ensuite faire place au « retour au village » de plusieurs exclus de la ville et à un regain de l'intérêt des élites extérieures pour le milieu rural.

Comparativement aux schémas organisationnels anciens, les GIC disposent de plus en plus d'un capital social qualitativement apprécié par les intervenants extérieurs ${ }^{(7)}$. En ce sens, l'ajustement des actions de recherche participative aux conditions sociales a été aisé. Par ailleurs, le déploiement des nouvelles organisations rurales (GIC, unions de GIC et fédérations d'unions de GIC) offre des canaux de propagation rapide des innovations agricoles. La dynamique organisationnelle est un élément favorable pour l'augmentation de l'impact de la recherche et de l'intervention. Dans certains cas, les institutions de recherche et les ONG cherchent à renforcer la création d'organisations de producteurs, en particulier lorsqu'elles ont besoin, pour la généralisation de leurs résultats et de leurs actions, de relais sociaux représentatifs. Ces formes organisationnelles, lorsqu'elles sont réellement efficientes, permettent également de rompre avec l'asymétrie des relations entre la recherche et l'intervention, d'une part, et les "élites ", d'autre part. Dans le domaine de la gestion des forêts, les conclusions provisoires des travaux en cours au Center for international forestry research (Cifor, Cameroun) révèlent que les comités de gestion des forêts communautaires et les comités de gestion des redevances forestières allouées aux communautés villageoises, calqués sur les modèles proposés par la réforme rurale (GIC, notamment), sont en inadéquation avec le fonctionnement des institutions locales.

Ainsi, l'Institut de recherche agricole pour le développement (Irad) au Cameroun et le Centre international de recherche agronomique pour le développement (Cirad) initient depuis quelques années des activités conjointes avec des organisations rurales. Ces activités portent sur la culture du palmier à huile, sur le maraîchage et sur la pisciculture, dans la mesure où les GIC sont plus ouverts à la spécialisation et à la professionnalisation agricole. Le Centre africain de recherches sur les bananiers et plantains (Carbap) met en place, en partenariat avec une quinzaine de GIC des 
provinces du Centre et du Sud, un réseau d'agriculteurs expérimentateurs. Dans la même logique, l'International Center for research in agro-forestry (Icraf), l'International Institute of tropical agriculture (IITA) et l'International Center for living aquatic ressource management (Iclarm) collaborent avec de nombreux GIC. Toutes les institutions de recherche tentent de construire et de coordonner des alliances avec les organisations rurales. Certains auteurs contestent la pertinence des organisations paysannes comme relais de la recherche pour la production d'innovations techniques (Floquet, 1994). En effet, les analyses sur les systèmes de production des connaissances révèlent une diversité de variables, dont principalement les facteurs culturels et ethniques qui s'ajoutent aux autres variables classiques de l'environnement macro et micro-économique. Les changements techniques seraient dès lors déterminés principalement par des interactions socio-économiques: "La manifestation des problèmes de technologie agricole constitue rarement un enjeu commun entre paysans " (Floquet, 1994, op. cit.). Dans certaines situations cependant, on constate que, si les objectifs des organisations collectives sont, à l'origine, d'ordre économique, social ou politique, ceux-ci deviennent rapidement des supports institutionnels pour la circulation de l'information et la dissémination des innovations technologiques (Temple et alii, 1994). L'instauration de connexions privilégiées entre la recherche agronomique et les dynamiques d'innovations endogènes est à l'origine de ces situations. La controverse sur ce sujet restera ici ouverte.

\section{Conclusion}

La réforme rurale camerounaise a été porteuse d'espoirs pour tous les acteurs du développement et de la recherche. Intervenue dans un contexte favorable de libéralisation du paysage sociopolitique et de décentralisation, elle a donné de nouvelles définitions à la société civile et à l'action communautaire. En un sens, elle a également ouvert des espaces de partenariat entre les organisations de producteurs, la recherche (agricole et forestière) et le développement. Les changements organisationnels que la réforme rurale a élaborés sont partie prenante d'un ensemble d'enjeux liés à la réadaptation des formes d'action de la recherche au nouveau contexte de pluralisme institutionnel et intellectuel en milieu rural. Cela exige une inversion du schéma traditionnel de relation entre la recherche, l'intervention et les bénéficiaires, en essayant autant que possible de voir en ces derniers des partenaires. Pour y arriver, la recherche et l'appui au développement agricole doivent faire appel à des méthodologies sociales adaptatives, fondées sur l'itération, la remise en question, la collaboration et l'apprentissage mutuel. Ce faisant, la recherche contribuerait à une structuration adéquate du partenariat pour le développement agricole. Elle rendrait aussi moins opaque la frontière entre les différents acteurs. 


\section{Bibliographie}

Alexandre P., 1965, « Protohistoire du groupe Béti-Fang, essai de systématisation ", Cahier d'études africaines, $\mathrm{n}^{\circ} 5$.

Amalric F., 1994, "Governance and Responsibility ", Development, 1994 : 1.

Beaudoux E., 1994, « Nouvelles pratiques d'appui aux organisations paysannes au Cameroun ", in Responsabilisation des agriculteurs en Afrique par le biais des organisations paysannes, AFTES document, The World Bank, Washington DC. Diaw M. C., 1997, Si, Nda Bot et Ayong: culture itinérante, occupation des sols et droits fonciers au Sud-Cameroun, Londres, ODI.

Engola Oyep J., 1997, « Les ONG après l'Etat en milieu rural au Cameroun ", in Cahiers de l'Ucac, 2, Yaoundé, pp. 177-185.

Floquet A., 1994, "Dynamique endogène du changement technique et organisation paysanne", in J.-P. Jacob et alii, Les associations paysannes en Afrique, Karthala.

Geshiere P., 1984, "La paysannerie africaine est-elle captive?", Politique africaine, $\mathrm{n}^{\circ} 14$.

Goyett H. et Kom J., 1993, Le cas de l'Amicale des amis II, la gestion d'une tontine, Montréal, Cetai.

Hartman E. H., 1987, «Some issues and priorities of the CGIAR in global agricultural research ", in Bonte-Fredheim C. et Sheridam K. (eds), The globalization of science, the place of agricultural research, new expanded edition, The Hague, Isnar.

Jarrige F., Touzard J.-M., 2001, « Les mutations de l'organisation coopérative à travers l'évolution de ses règles ", Revue internationale de l'économie sociale, $\mathrm{n}^{\circ} 280$, avril.

Lachenmann G., 1994, "Civil society and social movements in Africa ", in Jacob J.-P. et Lavigne Del Ville P. (édit.), Les associations paysannes en Afrique, organisation et dynamiques, Paris, Karthala.

Mercoiret M. R., 1990 «L'émergence des dynamiques sociales, une réponse au désengagement de l'Etat ", in L'avenir de l'agriculture dans les pays du Sahel, Montpellier, Cirad.

Moustier P., 1998, "Offre vivrière et organisation des échanges ", in Dossier de l'inter-réseaux: contrats et concertation entre acteurs des filières vivrières, mai 1998.

Oyono P. R., 2001, « Infrastructure organisationnelle et capacités locales dans la gestion décentralisée des forêts au Cameroun : éléments d'anthropologie écologique et leçons intermédiaires ", document du travail, YaoundéWashington DC, Cifor-WRI.

Russel D., 1993, "Resource management in Central African forest zone: a handbook for IITA's humid forest station ", report, Yaoundé, IITA.

Selener, D., 1997, Participary action research and social change, New York, CPARN-Cornell University.

Ssenkoloto G. M., 1984, « The psychology of participation in development work behaviour related to participation within the African context ", in Mondjanagui A. (édit.), La participation populaire en Afrique noire, Douala-Paris, IPD-Karthala.

Thrupp L. A., 1996, New partnership for sustainable agriculture, Washington DC, WRI.

Temple L., Touzard J.-M., Jarrige F., 1996, "La restructuration des coopératives vinicoles en Languedoc-Roussillon, du modèle communal à la diversité des adaptations actuelles ", Revue d'économie méridionale, vol 44, n 176, 4-1996, pp. 73-93. 